\title{
A novel insertion ins(18;5)(q21.1;q31.2q35.1) in acute myeloid leukemia associated with microdeletions at 5q31.2, 5q35.1q35.2 and $18 q 12.3 q 21.1$ detected by oligobased array comparative genomic hybridization
}

Eigil Kjeldsen

\begin{abstract}
Background: Nonrandom clonal chromosomal aberrations can be detected in approximately $55 \%$ of adult patients with acute myeloid leukemia (AML). Recurrent cytogenetic abnormalities play an important role in diagnosis, classification and prognosis of AML. However, several chromosomal abnormalities have not been completely determined or characterized, primarily because of their low incidence and limited amount of data.

Results: We characterized an AML patient with a novel apparently balanced insertion ins(18;5)(q21;q31.2q35.1) that was cryptic by G-banding. The rearrangement was further examined by molecular cytogenetic methods and oligobased high-resolution array CGH (oaCGH) analysis. We show that an approximately $31.8 \mathrm{Mb}$ large segment from chromosome 5 bands q31.2 to q35.1 has been inserted, by a direct mechanism, into chromosome 18 between bands q12.3 and q21.1. The insertion was unbalanced with concurrent submicroscopic deletions at 5q31.2 (approximately $0.37 \mathrm{Mb}$ in size), 5q35.1q35.2 (approximately $1.98 \mathrm{Mb}$ in size), and 18q12.3q21.1 (approximately 2.07 Mb in size). The microdeletions affect genes on $5 q$ and $18 q$ that have been associated with hematological malignancy and other cancers. A novel juxtaposition of the genes NPM1 and HAUS1 at 5q35.1 and 18q21.1, respectively, was detected by FISH analysis. Searching the literature and the Mitelman database revealed no previously reported ins $(18 ; 5)$ cases. Interestingly, however, two AML patients with translocation $t(5 ; 18)(q 35 ; q 21)$ encompassing the $5 q 35$ and 18 q21 breakpoint regions as detected in our present ins(18;5) patient have been reported.

Conclusions: It is well-known that cytogenetic abnormalities on the long arm of chromosome 5 affect hematopoiesis. However, the precise mechanism of their involvement in myeloid transformation is elusive. Our present data shed new light onto the frequent abnormalities on $5 q$ as well as to the less frequent abnormalities observed on $18 q$ in myeloid malignancies. In addition, we show that oaCGH analysis is a useful adjunct to revealing submicroscopic aberrations in regions of clinical importance. Reporting rare and nonrandom chromosomal abnormalities contribute to the identification of the whole spectrum of cytogenetic abnormalities in AML and their prognostic significance.
\end{abstract}

Keywords: Acute myeloid leukemia, ins(18;5), oaCGH analysis, Chromosomal insertion, Microdeletion, Cryptic chromosomal aberration, del(5q), add(18q)

Correspondence: Eigil.Kjeldsen@clin.au.dk

Department of Hematology, HemoDiagnostic Laboratory, Cancer Cytogenetics

Section, Aarhus University Hospital, Tage-Hansens Gade 2, Ent. 4A, DK-8000

Aarhus C, Denmark 


\section{Background}

In acute myeloid leukemia (AML) recurrent nonrandom chromosomal aberrations occur in approximately $55 \%$ of the patients. Until now about one hundred different chromosomal rearrangements have been uncovered in AML. The rearrangements mostly include balanced translocations, inversions, deletions, amplifications, monosomies and trisomies [1]. It is well established that cytogenetic analysis is an important prognostic factor that influences therapeutic decision-making and disease outcome because the various chromosomal rearrangements play critical roles in the molecular pathogenesis [2-4].

Myeloid malignancies are subdivided into distinct disease entities on the basis of specific cytogenetic or molecular genetic abnormalities [5]. Cytogenetic characterization defines three different risk groups: favorable, intermediate, and adverse [6]. Molecular characterization has revealed that mutations in FLT3 and NPM1 define molecular subgroups with prognostic relevance [7]. AML patients that do not fulfill WHO criteria for other categories are grouped together in the "AML, not otherwise specified (NOS)" category, which do not provide prognostic information. AML is a heterogeneous disease with respect to clinical and biological features. Hence, it is very important to better define less frequent chromosomal rearrangements in AML patients to identify the full spectrum of molecular prognostic factors.

Here we report the characterization of a novel cryptic insertion ins(18;5)(q21.1;q31.2q35.1) in a patient with de novo $\mathrm{AML}$, who, as detected by oligobased highresolution array CGH (oaCGH) analysis, also harbored three concurrent submicroscopic microdeletions 5q31.2, 5q35.1q35.2, and 18q12.3q21.1 in his leukemic cells. Two previous AML patients with the translocation $\mathrm{t}(5 ; 18)$ (q35; q21), and similar breakpoints as observed in our patient, have been reported. We review these patients and discuss the possibility that the ins $(18 ; 5)$ detected in our present patient is a variant of this rare non-random chromosomal abnormality $\mathrm{t}(5 ; 18)$.

\section{Case presentation}

A 37-year-old male Caucasian, previously well, presented with 4-5 weeks of fatigue, increasing paleness and dyspnea. In this period and on admission there were no febrilia, infections, or signs of bleeding except for one occasion of melaena 3 weeks prior to admission. He had an unintended weight loss of five $\mathrm{kg}$ from $91 \mathrm{~kg}$. Bone marrow (BM) examination showed marked hypercellularity with medium-sized mononuclear blasts and an $80 \%$ proportion of highly proliferative blasts, staining CD4+, CD7+, CD13+, CD43+, CD117+, CD123+, CD34-, HLA-DR+, CD56-, and TdT-. Hematological examination included a total white blood cell count of $4.49 \times 10^{9} / \mathrm{L}$, hemoglobin of $5.1 \mathrm{mmol} / \mathrm{L}$ and, platelets of $24 \times 10^{9} / \mathrm{L}$.
Segmented neutrophil count was $0.70 \times 10^{9} / \mathrm{L}$. The patient's father's cousin and great grandmother in his mother's line had leukemia. The patient had no comorbidity and had no previous history of being treated with chemotherapy or exposed to radiation. He had been smoking until 3 years prior to his AML diagnosis with an estimated pack years of 15 . There was no information on possible occupational hazards.

Our patient entered the AML-17 treatment protocol (Trial reference ISRCTN55675535). This protocol is a randomized multi-arm Phase III study designed by the AML Working Group of the National Cancer Research Institute (NCRI) and the Hematology Oncology Study Group in Acute Myeloid Leukemia and high risk Myelodysplastic Syndrome (MDS) in adults (http://aml17.cardiff. ac.uk/). In this interventional treatment protocol, AML and high risk MDS patients are randomized to one of five subgroups for induction therapy, then risk assessed, and randomized to FLT3 inhibitor if mutated or high risk chemotherapy with or without mTOR inhibition. According to the protocol our patient was initially treated with DA because of intermediate-risk cytogenetics. Molecular genetic analysis of his bone marrow cells at diagnosis showed an internal tandem duplication mutation in FLT3 (FLT3-ITD) and NPM1 $1^{\mathrm{wt}}$, and was then assigned to highrisk leukemia. He received FLAG-IDA treatment according to AML-17 and obtained complete remission 28 days after admission as evaluated by pathology, flowcytometry, cytogenetics and molecular genetics.

\section{Results}

\section{Cytogenetic and multicolor FISH analyses}

Karyotyping by G-banding of unstimulated cultured BM cells at initial diagnosis was interpreted as an apparently unbalanced male karyotype 46,XY,del(5)(q31q35),add(18) (q23)[25] (Figure 1A). To further characterize these chromosomal aberrations we next performed 24-color karyotyping using 24XCyte human multicolor FISH (mFISH) probe kit which revealed the insertion ins $(18 ; 5)$ and that there were no other structural abnormalities (Figure 1B). To further define the chromosome 5 segment that was cut out and where it was inserted into chromosome 18 we performed mBanding with XCyte probes for chromosomes 5 and 18 (Figure 2). These analyses showed that the chromosome segment 5q31q35 was inserted into chromosome 18 at band region $\mathrm{q} 21$ by a direct mechanism. By combining the obtained results a revised karyotype 46,XY, ins(18;5)(q21;q31q35)[25] could be made. Analysis of PHA-stimulated cultures of blood lymphocytes revealed a normal male karyotype, as did analysis of bone marrow cells after one induction series (data not shown). These data exclude the possibility that the observed ins $(18 ; 5)$ in the patient's bone marrow cells at diagnosis was constitutional. 


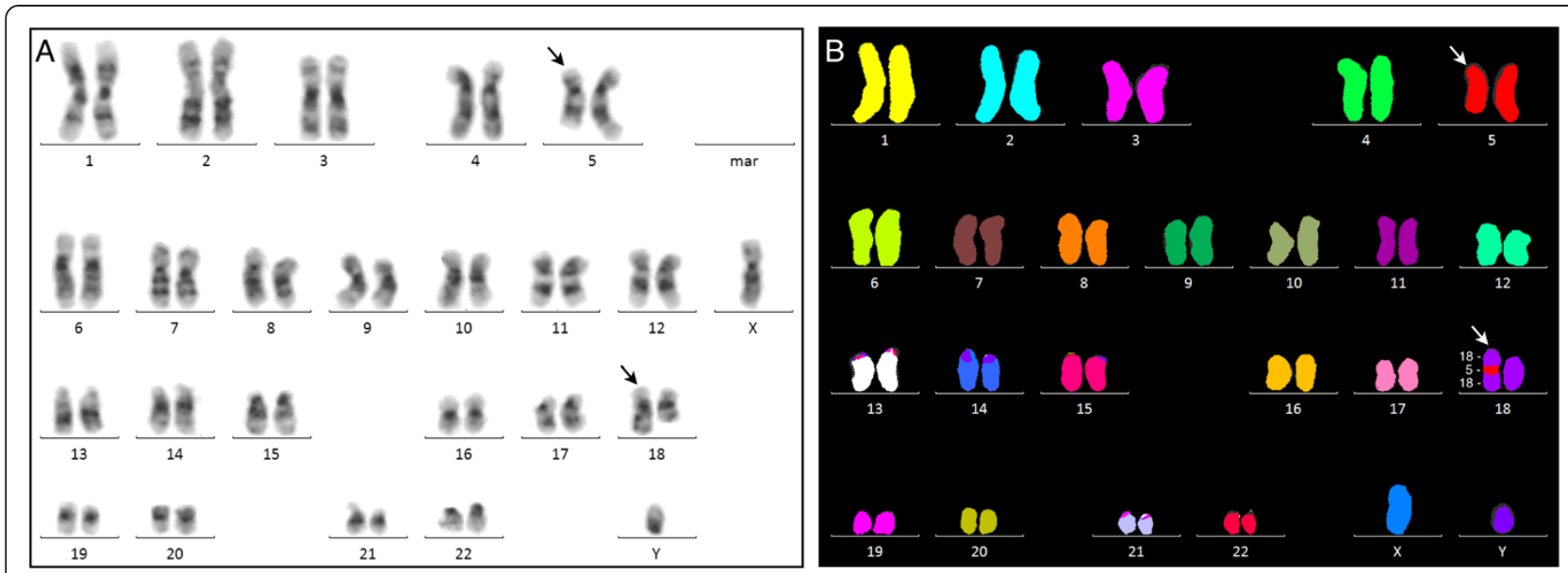

Figure 1 Karyotyping analyses. A. G-banding analysis showed an aberrant karyotype initially interpreted as 46,XY,del(5)(q22q35),add(18)(q23)[25]. B. 24-color karyotyping revealed the cryptic insertion ins(18;5)(q21;q31q35) in all 10 analyzed metaphases. Arrows indicate the aberrant chromosomes.

\section{oaCGH analysis}

To search for possible copy number abnormalities involved in the ins $(18 ; 5)$ rearrangement we performed oaCGH analysis using the CytoChip Cancer $4 \times 180 \mathrm{~K}$ v2.0 (BlueGnome, Cambridge, UK). The oaCGH analysis detected four somatic copy number alterations in the form of three microdeletions at 5q31.2, 5q35.1-q35.2 and 18q12.3-q21.1 (Figure 3), and a single microamplification at $12 \mathrm{q} 21.1$. The microamplification had a maximal size of $158.3 \mathrm{~kb}$ (pos. 72,596,354-72,754,669) (Max: A_16_ P19594168: 72,596,354 to A_16_P19594509: 72,754,669) and a minimum size of $107.0 \mathrm{~kb}$ (Min: A_16_P02650455: 72,625,008 to A_16_P19594474: 72,732,027) but this region contains no known genes (data not shown).

The maximum regions of microdeletions involved in the other break point regions are: 1) at chromosome band $5 \mathrm{q} 31.2$ the microdeletion encompasses the oligonucleotide probes A_16_P37384388 to A_16_P17320593 mapping from $138,390,821$ to $138,769,054 ; 2$ ) at chromosome bands 5q35.1-q35.2 the microdeletion encompasses the oligonucleotide probes A_16_P37464643 to A_16_P17402538 mapping from 170,768,753 to $172,758,763$; and 3) at chromosome bands 18q12.3-q21.1 the microdeletion encompasses the oligonucleotide probes A_16_ P41020231 to A_16_P03359511 mapping from 39,887,338 to $41,970,952$. The minimum region of microdeletions in the involved break point regions are: 1) at chromosome band $5 \mathrm{q} 31.2$ the microdeletion encompasses the oligonucleotide probes A_16_P17319737 to A_16_P17320525 mapping from $138,403,448$ to $138,746,932 ; 2$ ) at chromosome bands 5q35.1-q35.2 the microdeletion encompasses the oligonucleotide probes A_18_P15493901 to A_16_ P01397609 mapping from 170,771,533 to $172,741,295$; and 3 ) at chromosome bands $18 q 12.2-q 21.1$ the microdeletion encompasses the oligonucleotide probes A_16_P20842291 to A_16_P20847704 mapping from 39,899,537 to $41,952,135$. From these results the respective estimated minimum to maximum deletion sizes are: 1) at $5 \mathrm{q} 31.2$ : 343.5-378.2 kb; 2) at 5q35.1-q35.2: 1,969.8-1,990.0 kb; and 3) $18 \mathrm{q} 12.3-\mathrm{q} 21.1: 2,052.6-2,083.6 \mathrm{~kb}$. The genes located in the minimal deleted regions are summarized in Figure 3.

\section{FISH analyses}

To validate the microdeletions, FISH analyses were performed using several BAC-based custom made probes. These were co-hybridized with subtelomeric probes from 5qter and 18qter, and compared to dual color whole chromosome painting with probes for chromosomes 5 and 18 and FISH analysis with the EGR1(5q31)/ D5S23,D5S721(5p15.2) dual color probe (Figure 4A). The experiments showed that: 1) the EGR1 gene is not part of the proximal microdeletion as expected from the oaCGH analysis; 2) the BAC-based probes RP11-118P24 (5q31.2), RP11-145P20 (5q35.1) and RP11-9H20 (18q12.3) all showed mono-allelic deletions confirming the microdeletions as suggested by the oaCGH analysis; and 3) the microdeletions on $5 \mathrm{q}$ are located on the same short derivative homologue of chromosome 5. Counting 200 interphase nuclei using each of the BAC-probes showed that approximately $90 \%$ of the interphase nuclei contained the microdeletions. Using the BAC-probe RP11-184C9 (5q35.1) it was confirmed that it is not part of the deleted region, as expected from the oaCGH analysis, but was part of the $5 \mathrm{q}$ fragment that was inserted onto chromosome 18. Analyzing 200 interphase nuclei with this probe a normal signal pattern of 2R2G was observed in all of the examined cells, confirming that this probe is not part of the deleted region.

Taken together we have shown that an approximately 31.8 Mb large chromosomal segment encompassing the 


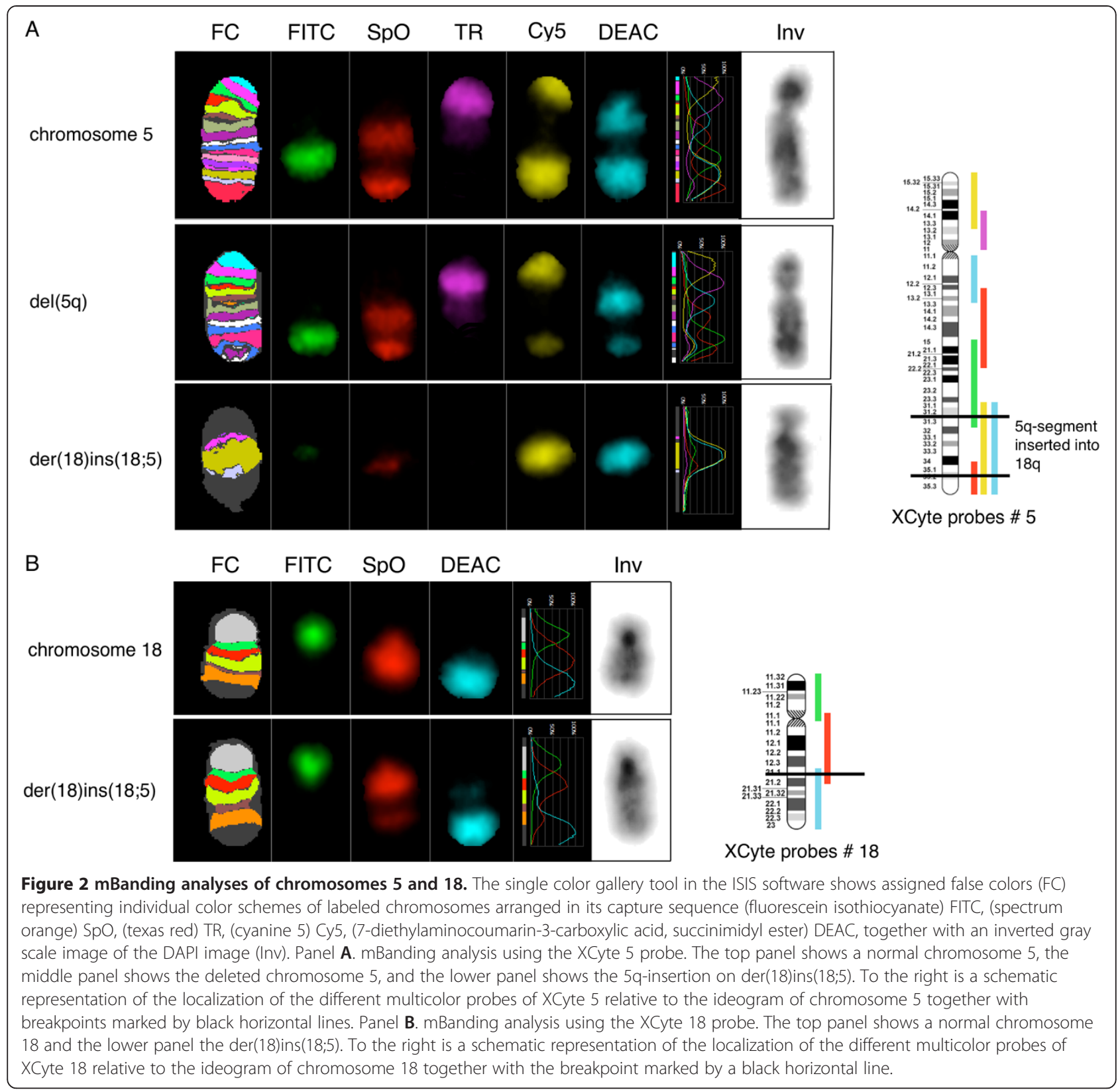

bands $5 \mathrm{q} 31.2 \mathrm{q} 35.2$ was cut out and inserted by a direct mechanism between chromosome bands q12.3 and q21 on chromosome 18 (Figure 4B and C). The insertion was unbalanced with concurrent submicroscopic deletions at $5 \mathrm{q} 31.2$ (approximately $0.37 \mathrm{Mb}$ in size), 5q35.1q35.2 (approximately $1.98 \mathrm{Mb}$ in size), and 18q12.3q21.1 (approximately $2.07 \mathrm{Mb}$ in size). As a result of this complex rearrangement the following band regions became juxtaposed: 5q31.2-5q35.2, 18q12.3-5q31.2, and 5q35.118q21.1. By in silico analysis of the involved regions it was found that the $5^{\prime}$-part of SIL1 (spanning exons 19) at 5q31.2 and the $5^{\prime}$-part of HAUS1 (spanning exons 1-4) were deleted. A fusion of the chromosomal regions $5 \mathrm{q} 35.1$ and $18 \mathrm{q} 21.1$ spanning the NPM1 and HAUS1 genes, respectively, was confirmed by FISH analysis with the RP11-117L6 and RP11-474O19 probes (Figure 4D).

\section{Discussion}

The insertion ins(18;5)(q21.1;q31.2q35.1) detected in the leukemic cells of the presented de novo AML patient is to the best of our knowledge a novel chromosomal abnormality. A systematic review of the literature and a search in the Mitelman database [1] did not reveal any previous reports on ins $(18 ; 5)$ patients with hematological or other cancers. 


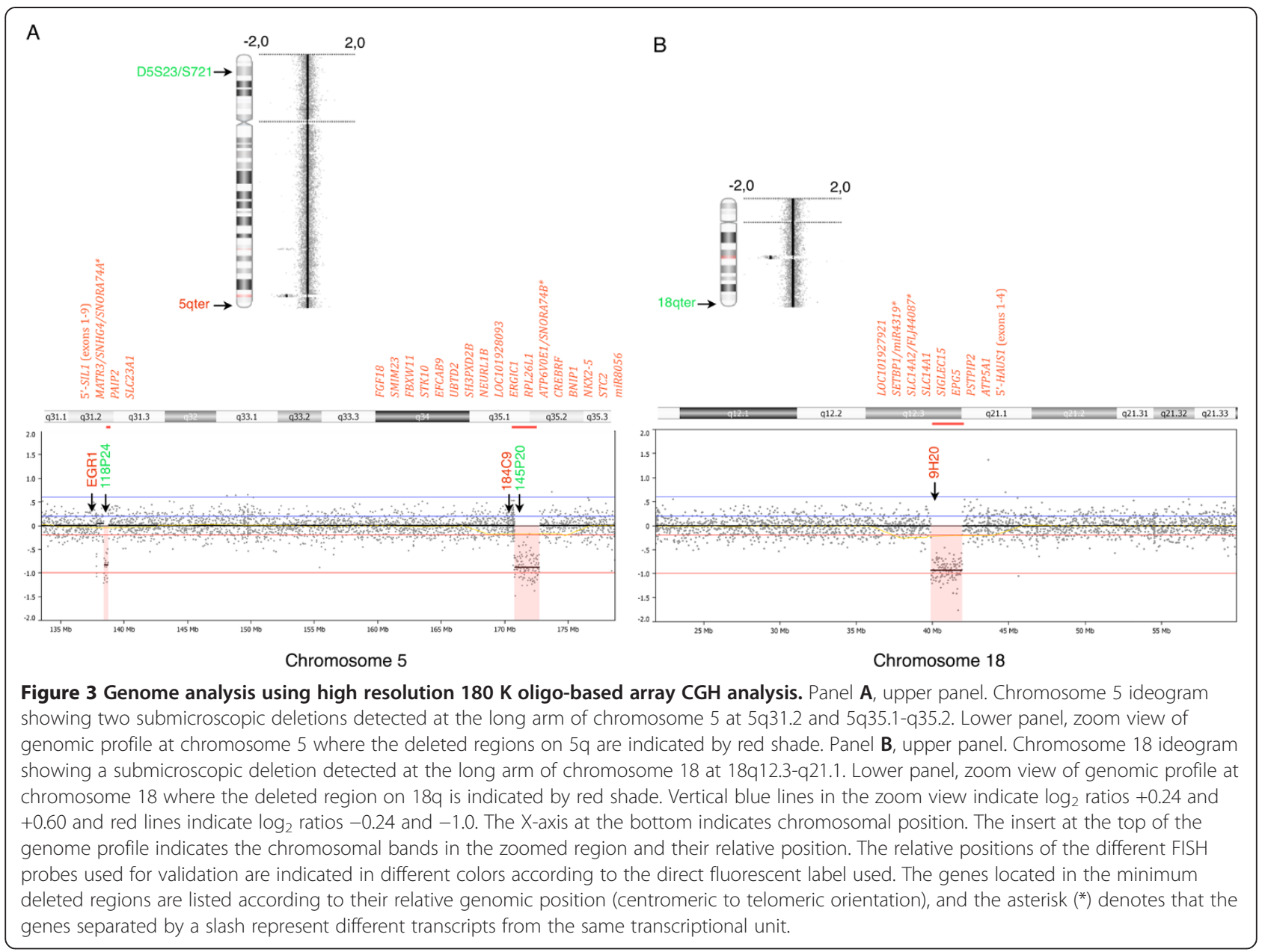

Insertions are very rare chromosomal abnormalities, not only in hematological malignancies but also in constitutional genetics where the incidence was estimated to be as low as 1:80,000 [8]. Despite their rarity, several instances of insertion variants of the traditional common translocations have been reported in myeloid malignancy, including ins $(8 ; 21) /$ ins $(21 ; 8)$ and $\mathrm{t}(8 ; 21)(\mathrm{q} 22 ; \mathrm{q} 22) /$ RUNX1-RUNXT1 [9], ins(3;5) and $\mathrm{t}(3 ; 5)(\mathrm{q} 25 ; \mathrm{q} 35) / N P M 1-$ $M L F 1$ [10], and ins(22;9) and $\mathrm{t}(9 ; 22)(\mathrm{p} 24 ; \mathrm{q} 11.2) / B C R-$ $J A K 2$ [11]. Although the molecular mechanisms for generating the insertions variants are different compared to those of generating the traditional common translocations the insertion variants have similar aberrant fusion genes. Further, the AML patients with the insertion variants exhibit similar morphology and prognosis compared to their traditional translocation counterparts.

These observations prompted us to speculate whether the rearrangement observed in our patient could be a variant of a putative $t(5 ; 18)$. Interestingly, a revised search revealed two previously reported AML patients with translocation $\mathrm{t}(5 ; 18)(\mathrm{q} 35 ; \mathrm{q} 21)[12,13]$ involving the same cytogenetic bands as in our patient. The clinical and genetic findings are summarized in Table 1 . No additional cases could be found after a search in our cytogenetic registry containing more than 2,400 sequential entries of AML since 1990. Although all three patients share similar cytogenetic break points, the rearrangements may still be very different at the molecular level. Since there are no information regarding molecular breakpoint mapping or studies of possible concurrent submicroscopic aberrations in the reported $t(5 ; 18)$ patients this question cannot be addressed. From a clinical point of view it was remarkable that both patients were AML FAB subtype M2 and considered high-risk patients. For patient 2 this was because of an accompanying FLT3-ITD mutation while the basis for this assignment in patient 1 was cryptic. Our patient had AML with FAB subtype M1 and considered high-risk because he had an accompanying FLT3-ITD mutation. Patient 1 had bone marrow transplantation (BMT) in first remission. A BMT in CR1 is planned in our patient but this has been postponed because of complicating fungal infections.

Together these observations suggest that $t(5 ; 18)$, and perhaps our possible variant ins(18;5), may be associated 


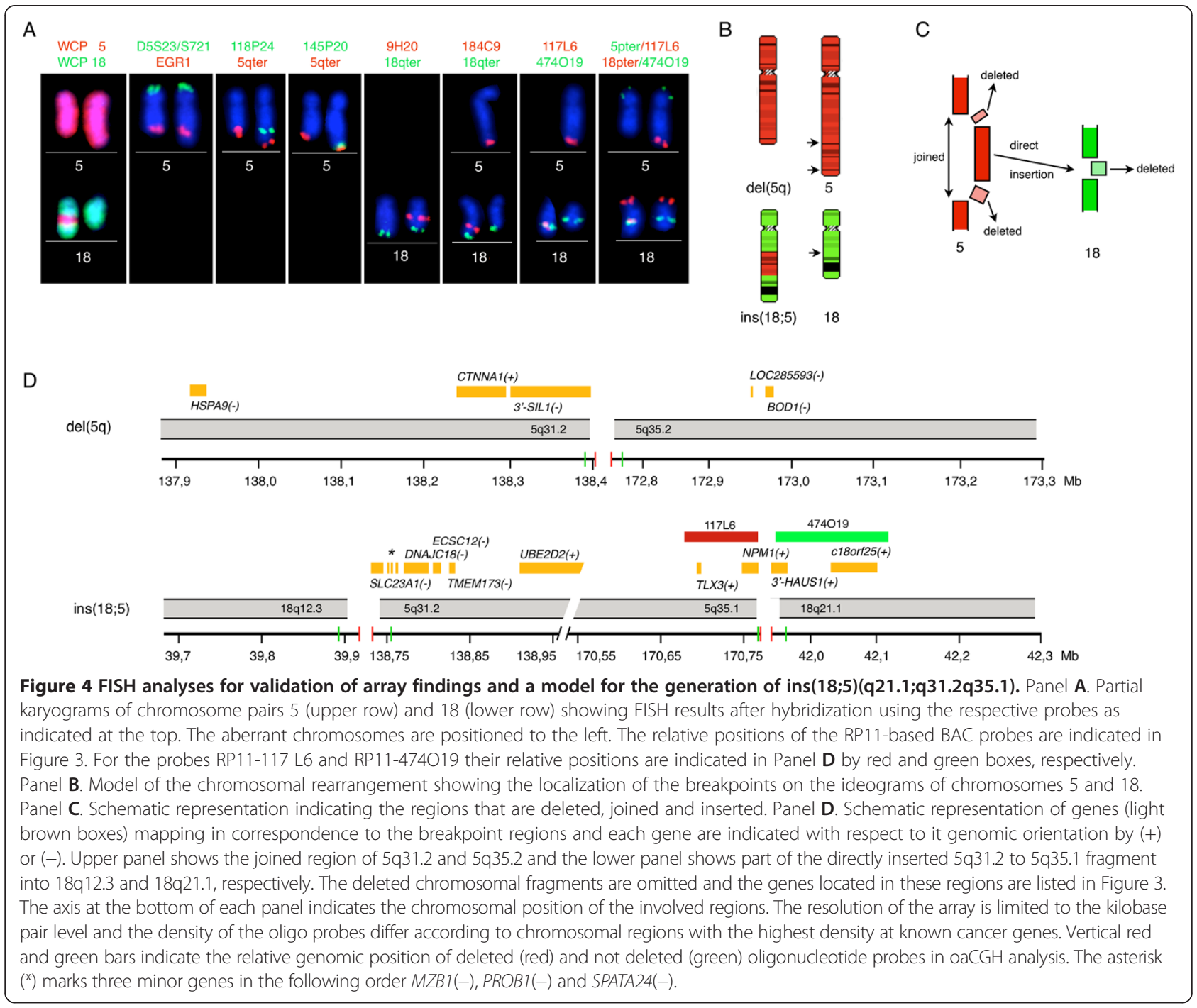

with a high risk AML FLT3-ITD subgroup although the contribution of each genotypic component is unknown. It is well known, however, that AML patients with a normal karyotype and a high burden of FLT3-ITD often present with a more aggressive disease; and more often relapse after remission [14-16]. The impact of FLT3-ITD among other cytogenetic subgroups is not clear $[15,17]$.

With the aid of oaCGH analysis we detected concurrent submicroscopic deletions at each of the cytogenetic breakpoints involved in the complex rearrangement, including 5q31.2, 5q35.1q35.2 and 18q12.3q21.1. Submicroscopic deletions surrounding the most common recurrent translocations breakpoints have been reported in various leukemia with incidences ranging from approximately $2 \%$ to $20 \%$, including $\mathrm{t}(8 ; 21)(\mathrm{q} 22 ; \mathrm{q} 22), \mathrm{t}(9 ; 22)$ (q34; $\mathrm{q} 11)$, and $\mathrm{t}(15 ; 17)(\mathrm{q} 22 ; \mathrm{q} 22)$ [18-21]. The clinical significance of these accompanying submicroscopic deletions is largely unknown because they in some cases were associated with poor therapy response and unfavorable outcome while they in others had no effect. It was hypothesized that deletion of critical genes could account for the possible difference in disease course, or alternatively, that such deletions reflect an underlying genomic instability that may predispose the malignant cells to acquire other genetic abnormalities that confer a worse prognosis.

Simple reciprocal translocations between two nonhomologous chromosomes fundamentally require only two chromosomal double stranded DNA breaks (DSBs) followed by an exchange of the resulting fragments before sealing of the breaks. A simple insertion requires three chromosomal DSBs, transfer of the segment and then sealing of the three breaks. When a simple insertion rearrangement is complicated by accompanying submicroscopic deletions in the kilobase-to-megabase size at each of the breakpoints, as described in our patient (Figure $4 \mathrm{~B}$ and $4 \mathrm{C}$ ), at least six DSBs are required for the complex rearrangement to occur. The additional submicroscopic deletions could either be a by-product 
Table 1 Summary of published AML patients with $t(5 ; 18)(q 35 ; q 21)$ and present patient with ins(18;5)(q21;q31q35)

\begin{tabular}{|c|c|c|c|}
\hline Reference & Patient 1 [12] & Patient 2 [13] & Present case \\
\hline Age (yr) & 42 & 73 & 37 \\
\hline Gender & Female & Male & Male \\
\hline WBC $\left(\times 10^{9} / L\right)$ & 48.1 & 12.0 & 4.5 \\
\hline Platelets $\left(\times 10^{9} / \mathrm{L}\right)$ & 56 & 65 & 24 \\
\hline $\mathrm{Hgb}(\mathrm{mM})$ & $6.0^{\mathrm{a}}$ & $7.2^{\mathrm{a}}$ & 5.1 \\
\hline $\begin{array}{l}\text { BM Morphology: Blast (\%), } \\
\text { cell size, cellularity }\end{array}$ & $80 \%$, large size, hypocellular & $60 \%$, large size & $80 \%$, medium size, hypercellular \\
\hline Immunophenotype & $\begin{array}{l}\text { CD4+, CD13+, CD33+, HLA-DR+, } \\
\text { CD38+, CD11c+, CD117+ }\end{array}$ & CD13+, CD33+, HLA-DR+, CD117+, MPO- & $\begin{array}{l}\text { CD4+, CD7+, CD13+, CD33+, CD117+, } \\
\text { MPO+, CD56-, CD34-, TdT- }\end{array}$ \\
\hline Diagnosis & AML-M2 (de novo) & AML-M2 (de novo) & AML-M1 (de novo) \\
\hline Cytogenetics & $46, X X, t(5 ; 18)(q 35 ; q 21)[14]$ & $46, X Y, t(5 ; 18)(q 35 ; q 21)[2] / 46, X Y[12]$ & $46, X Y$, ins $(18 ; 5)(q 21.1 ; q 31.2 q 35.1)[25]$ \\
\hline \multirow[t]{4}{*}{ aCGH findings } & No information & No information & 0.16 Mb amplification @12q21.1 \\
\hline & & & 0.37 Mb deletion @5q31.2 \\
\hline & & & 1.98 Mb deletion @5q35.1q35.2 \\
\hline & & & 2.07 Mb deletion @18q12.3q21.1 \\
\hline Gene Mutations & No information & $F L T 3^{\mid T D}$ & $F L T 3^{\mid T D}, N M P 1^{w t}$ \\
\hline \multirow[t]{3}{*}{ Outcome } & BMT in CR1 & Obtained CR1 & Obtained CR1, karyotype 46,XY[25], \\
\hline & & $\begin{array}{l}\text { Relapse with } \mathrm{t}(5 ; 18)(\mathrm{q} 35 ; \mathrm{q} 21) \text { and } \\
\text { a secondary } \mathrm{t}(3 ; 12)(\mathrm{p} 23 ; \mathrm{p} 13)\end{array}$ & BMT planned in CR1 \\
\hline & & Died 18 months after initial diagnosis & \\
\hline
\end{tabular}

Published values converted to SI units.

of the chromosomal repair mechanism or part of an initiating event. The major DSB repair pathways in mammalian cells are the homologous recombination (HR) and non-homologous end-joining (NHEJ) pathways, and depending upon the chosen repair pathway aberrant chromosomal rearrangements can be generated [22]. In our patient it is likely that the error-prone NHEJ pathway repaired the respective breakpoints.

Translocations involving chromosome 5q35 are rare clonal abnormalities in hematological cancers [23]. The most common recurrent $5 \mathrm{q} 35$ translocations with formation of aberrant fusion genes are: $\mathrm{t}(2 ; 5)(\mathrm{p} 23 ; \mathrm{q} 35) /$ NPM1-ALK in anaplastic large cell lymphoma [24], $\mathrm{t}(3 ; 5)$ (q25; 35$) / N P M 1-M L F 1$ in AML [10], t(5;17)(q35; $\mathrm{q} 21) / N P M 1-R A R A$ in APL [25], in $\mathrm{t}(5 ; 11)(\mathrm{q} 35 ; \mathrm{p} 15) /$ NSD1-NUP98 in childhood AML [26], and $\mathrm{t}(5 ; 11)$ (q35;q12)/NSD1-FEN1 in AML-M5 [27]. The NPM1 and NSD1 at $5 \mathrm{q} 35$ are common translocation partners. The NSD1 gene encodes a nuclear protein involved in transcriptional regulation. No prognostic mutations have been ascribed to this gene in relation to leukemia. The NPM1 gene encodes a nuclear matrix phosphoprotein involved in nucleolar ribosome assembly and protein localization. In addition to being a translocation partner NPM1 can be affected by mutations at the DNA sequence level. A 4 bp insertion in exon 12 in NPM1 is one of the most frequent genetic changes known in AML patients with a normal karyotype AML; and its presence in those patients confers a better prognosis [28]. In our patient we detected no genetic abnormalities in NPM1 except for its juxtaposition to HAUS1 at $18 \mathrm{q} 21.1$. In the previously reported AML patients with $\mathrm{t}(5 ; 18)(\mathrm{q} 35 ; \mathrm{q} 21)$ there is no information about possible aberrant fusion genes or concurrent additional abnormalities involving the NPM1 or NSD1 at 5q35.

Chromosomal abnormalities of the long arm of chromosome 18 are most often associated with lymphoid malignancies. However, the number of reports of genes on chromosome 18 involved in myeloid malignancy is increasing. The SETBP1 and its intronic MIR4319 at $18 \mathrm{q} 12.3$ were recently described to be new players in myeloid malignancy $[29,30]$. SETBP1 was shown to be overexpressed in secondary AML patients bearing the $\mathrm{t}$ $(12 ; 18)(\mathrm{p} 13 ; \mathrm{q} 12)$ while the intronic MIR4319 was downregulated [30]. Although the function of SETBP1 is unknown it has been implicated as a transcriptional regulator of many genes. Recurrent somatic mutations promote leukemic cell proliferation [29] and appear to be a poor prognostic marker especially in elderly AML patients [31]. In our AML patient we found that eleven RefSeq at $18 \mathrm{q} 12.3 \mathrm{q} 21.1$ were completely or partially deleted (Figure 3), and that the SETBP1 and MIR4319 genes were two of the deleted genes. In addition, we found that the $5^{\prime}$-part of HAUS1 at 18q21.1 was partially deleted and that it has become juxtaposed to NPM1 as a result of the complex insertion-deletion rearrangement. 
However, with the methods we used we cannot establish whether the NPM1 and HAUS1 genes formed an aberrant fusion gene. HAUS1 encodes one of eight subunits of the $390 \mathrm{kDa}$ human augmin complex that is a microtubule-binding complex vital for mitotic spindle assembly [32]. There are no previous reports on chromosomal rearrangements involving this gene.

Monosomy 5 and interstitial deletions of $5 \mathrm{q}$ are common chromosomal abnormalities in myeloid malignancy. These aberrations occur in 5-10\% of karyotypic abnormal adult AML and are usually associated with complex karyotypes, rapid disease progression and poor outcome $[24,33]$. In our patient we detected two interstitial microdeletions at $5 \mathrm{q}$, one at $5 \mathrm{q} 31.2$ (between 343,5$378,2 \mathrm{~kb}$ in size) and another at 5q35.1-q35.2 (between $1.969,8-1.990,0 \mathrm{~kb}$ in size). The minor $5 \mathrm{q} 31.2$ deletion overlaps with the centromeric commonly deleted region (CDR) of two previously identified CDRs in myeloid malignancies [33-35]. Of the CDRs the centromeric CDR at $5 \mathrm{q} 31.1-\mathrm{q} 32.2$ is common in high risk MDS and in AML, while the telomeric CDR at $5 \mathrm{q} 33.1$ is associated with $5 \mathrm{q}$ syndrom. The identification of pathogenic genes on $5 \mathrm{q}$ has proven to be challenging because most patients have extensive deletions encompassing both CDRs [36]. In a large SNP-based study it was found that among 1,115 examined patients with myeloid malignancies $12 \%$ had $5 \mathrm{q}$ deletions with a median size of 71,4 $\mathrm{Mb}$ ranging from $1.9 \mathrm{Mb}$ to $131.28 \mathrm{Mb}$ (whole arm) [37].

Since no single gene on $5 \mathrm{q}$ has been proven to be responsible for high risk myeloid malignancies a haploinsufficiency model has been proposed, reviewed in [33]. According to this model, loss of a single allele of more than one gene on $5 \mathrm{q}$ may act in concert to alter hematopoiesis, promote self-renewal of hematopoietic stem and progenitor cells (HSPCs), induce apoptosis of hematopoietic cells, and disrupt differentiation $[38,39]$. A number of candidate tumor-suppressor genes located at the centromeric CDR at $5 \mathrm{q} 31$ (including $C D C 25 C$, EGR1, HSPA9, CTNNA1, and DIAPH1) have been implicated in the development of high risk MDS/AML. In our patient we found that six and seventeen RefSeq genes at 5q31.2 and 5q35.1q35.2, respectively, were completely or partially deleted as illustrated in Figures 3 and 4D. It is noticed that the alpha-1 E-catenin gene, CTNNA1, at $5 \mathrm{q} 31.2$ is not involved in copy number alterations while the $5^{\prime}$-part of the downstream neighbor gene SIL1 is deleted. The CTNNA1 gene is a tumor suppressor gene that has been associated with progression and poor prognosis in leukemia [40]. The SIL1 gene has not previously been reported to be directly involved in myeloid malignancy although it often is one of the many genes that are deleted in high risk AML/MDS patients with del(5q). It encodes a nucleotide exchange factor that is important for the function of glucose-regulated protein 78 (GRP78). GRP78 is known as a stressinducible endoplasmic reticulum (ER) chaperone protein and serves as a master initiator of ER stress signaling [41]. Accumulation of unfolded proteins results in GRP78 activation via SIL1 and subsequently activation of the unfolded protein response (UPR). Proteasome inhibitors, such as bortezomib, suppress the degradation of unfolded proteins and trigger ER stress leading to activation of UPR and subsequently apoptotic signals. Although bortezomib is mainly used for treatment of multiple myeloma [42] and mantle cell lymphoma [43] it has in some instances been shown to be an effective agent for treatment of 5q- MDS [44]. The combination of genes that are completely or partially deleted in myeloid malignancies with interstitial deletions of $5 \mathrm{q}$ might contribute to the heterogeneity of high risk AML/MDS patients.

Application of array-based CGH analysis has not only significantly improved the detection rate of chromosome aberrations in patients with hematological malignancy compared to traditional cytogenetics [45-47] but also uncovered concurrent microdeletions in patients with apparently balanced translocations $[21,45]$. The present study, add to the knowledge of chromosomal aberrations and indicate that oaCGH is a useful adjunct to revealing submicroscopic aberrations in genomic regions of clinical importance.

\section{Conclusions}

The present study characterizes a high-risk de novo AML patient and reports on a novel rather complex insertion ins(18:15)(q21;q31.2q35.1) with concurrent submicroscopic deletions at 5q31.2, 5q35.1q35.2 and $18 \mathrm{q} 12.3 \mathrm{q} 21.1$. The rearrangement might be a variant of the chromosomal translocation $\mathrm{t}(5 ; 18)$ (q35; 21$)$, which previously was reported in two cases with high-risk de novo AML. This study also highlights the clinical usefulness of oaCGH analysis to identify additional submicroscopic copy number aberrations. We have narrowed the $5 q 31.2 \mathrm{CDR}$ in AML and provided new insight to the putative role of the $5 \mathrm{q} 31.2$ deletion in myeloid malignancy. In addition, we have uncovered a novel fusion of

Table 2 Summary of custom made BAC-based probes for characterization and validation of $\mathrm{OaCGH}$ findings

\begin{tabular}{lll}
\hline BAC probe & Cytoband & Genomic position (bp) \\
\hline RP11-118P24 & $5 q 31.2$ & $138,473,339-138,673,394$ \\
RP11-184C9 & $5 q 35.1$ & $170,301,198-170,446,174$ \\
RP11-117L6 & $5 q 35.1$ & $170,679,528-170,854,638$ \\
RP11-145P20 & $5 q 35.1$ & $170,858,901-171,048,742$ \\
RP11-9H20 & $18 q 12.3$ & $40,480,006-40,631,549$ \\
RP11-474019 & $18 q 21.1$ & $41,949,215-42,121,248$
\end{tabular}

${ }^{\mathrm{a}}$ Genomic position are given according to NCBI build 36.1 (hg18). 
the chromosomal regions at $5 \mathrm{q} 35$ and $18 \mathrm{q} 21.1$ containing the genes NPM1 and HAUS1, respectively, as a result of the complex insertion-deletion rearrangement. This study contributes to the identification of the whole spectrum of cytogenetic abnormalities in AML and their prognostic significance.

\section{Methods}

\section{G-banding analysis}

Chromosome analysis was done on G-banded chromosomes prepared after short-term unstimulated culturing of cells obtained from bone marrow at diagnosis, and Gbanding performed on PHA-stimulated peripheral blood cells as described [48]. Karyotypes were described according to ISCN [49].

\section{Fluorescent in situ Hybridization (FISH) analysis}

Human multicolor FISH were done according to manufacturer's instructions using the following XCyting multicolor FISH probes: 1) 24-color karyotyping was done with the 24XCyte consisting of 24 different chromosome painting probes, 2) mBanding with XCyte 5 and XCyte 18 probes consisting of a series of partial chromosome paints for sequential partially overlapping chromosome regions of a single chromosome (MetaSystems, Altlussheim, Germany). Each of the XCyte probes was labeled with one of five fluorochromes or a unique combination thereof (combinatorial labeling). Metaphases were counterstained with 4',6-diamidino-2-phenylindole (DAPI). Image capture was done with an automated Zeiss Axio Imager.Z2 equipped with a CCD-camera (CoolCube1) and appropriate filters using Isis software (MetaSystems). Karyotyping was done using the 24-color mFISH upgrade package, ISIS, including mBanding.

Whole chromosome painting and locus specific FISH analysis was done with the following directly labeled probes according to manufacturers' instructions: 1) whole chromosome painting probes for chromosomes 5 and 18 (Kreatech Diagnostics, Amsterdam, The Netherlands); 2) the LSI EGR1(5q31)/D5S23,D5S721(5p15.2) dual color probe set (Abbott Molecular, Wiesbaden, Germany); and 3) subtelomeric probes for 5pter, 5qter, 18 pter and 18 qter (Kreatech Diagnostics). Table 2 summarizes the custom made BAC-based probes (Empire Genomics, New York, USA) for validating the oaCGH findings. Chromosomes were counterstained with DAPI. FISH results were reported according to ISCN [49].

\section{Oligobased array comparative genomic hybridization analysis}

oaCGH analysis was performed using CytoChip Cancer $4 x 180 K$ v2.0 (BlueGnome, Cambridge, UK) encompassing a $20 \mathrm{~kb}$ backbone with highest concentration of probes at 670 cancer genes. The analysis was done according to manufacturer's instructions using $0.5 \mu \mathrm{g}$ patient DNA from bone marrow cells at initial diagnosis as described in [48]. After hybridization, washing and drying the oligo array was scanned at $2.5 \mu \mathrm{m}$ with GenePix 4400A microarray scanner. Initial analysis and normalization was done with BlueFuseMulti v2.6. For analysis and visualization normalized $\log 2$ probe signal values were imported into Nexus Copy Number software v. 6.1 (BioDiscovery, California, USA) and segmented using FASST2 segmentation algorithm with a minimum of 3 probes/segment. Regions of gain or loss contained within copy number variable regions $(\mathrm{CNVs})$ were discarded. Reference genome was NCBI build 36.1 (hg18). Bioinformatics analysis was performed by querying the UCSC database (http://genome.ucsc.edu).

\section{Consent}

The study conforms to the provisions of the Declaration of Helsinki. Written informed consent was obtained from the patient. A copy of the written consent is available for review by the Editor-in-Chief of this journal.

\section{Abbreviations}

BAC: Bacterial artificial chromosomes; PHA: Phytohemagglutinin; WHO: World Helath Organization.

\section{Competing interests}

The author declares that he has no competing interest.

\section{Acknowledgements}

The biotechnologists Bente Madsen and Pia Kristensen are greatly thanked for excellent technical assistance. The Danish Cancer Society supported the study.

Received: 18 July 2014 Accepted: 28 August 2014

Published online: 25 September 2014

\section{References}

1. Mitelman F, Johansson B, Mertens FE: Mitelman Database of Chromosome Aberrations and Gene Fusions in Cancer (2014). Last update: May 13, 2014. http://cgap.nci.nih.gov/Chromosomes/Mitelman.

2. Kayser S, Zucknick M, Dohner K, Krauter J, Kohne CH, Horst HA, Held G, von Lilienfeld-Toal M, Wilhelm S, Rummel M, Germing U, Gotze K, Nachbaur D, Schlegelberger B, Gohring G, Spath D, Morlok C, Teleanu V, Ganser A, Dohner H, Schlenk RF, German-Austrian AMLSG: Monosomal karyotype in adult acute myeloid leukemia: prognostic impact and outcome after different treatment strategies. Blood 2012, 119:551-558.

3. Grimwade D, Mrozek K: Diagnostic and prognostic value of cytogenetics in acute myeloid leukemia. Hematol Oncol Clin North Am 2011, 25:1135-1161. vii.

4. Mrozek K, Heinonen K, Bloomfield CD: Clinical importance of cytogenetics in acute myeloid leukaemia. Best Pract Res Clin Haematol 2001, 14:19-47.

5. Vardiman JW, Thiele J, Arber DA, Brunning RD, Borowitz MJ, Porwit A, Harris NL, Le Beau MM, Hellstrom-Lindberg E, Tefferi A, Bloomfield CD: The 2008 revision of the World Health Organization (WHO) classification of myeloid neoplasms and acute leukemia: rationale and important changes. Blood 2009, 114:937-951.

6. Grimwade D, Hills RK, Moorman AV, Walker H, Chatters S, Goldstone AH, Wheatley K, Harrison CJ, Burnett AK: Refinement of cytogenetic classification in acute myeloid leukemia: determination of prognostic significance of rare recurring chromosomal abnormalities among 5876 younger adult patients treated in the United Kingdom Medical Research Council trials. Blood 2010, 116:354-365.

7. Estey EH: Acute myeloid leukemia: 2013 update on risk-stratification and management. Am J Hematol 2013, 88:318-327. 
8. Van Hemel JO, Eussen $\mathrm{HJ}$ : Interchromosomal insertions. Identification of five cases and a review. Hum Genet 2000, 107:415-432.

9. Rucker FG, Bullinger L, Gribov A, Sill M, Schlenk RF, Lichter P, Dohner $H$, Dohner K: Molecular characterization of AML with ins(21;8)(q22;q22q22) reveals similarity to t(8;21) AML. Genes Chromosomes Cancer 2011, 50:51-58.

10. Arber DA, Chang KL, Lyda MH, Bedell V, Spielberger R, Slovak ML: Detection of NPM/MLF1 fusion in t(3;5)-positive acute myeloid leukemia and myelodysplasia. Hum Pathol 2003, 34:809-813.

11. Xu Y, Yin J, Pan J, Wu C, Wang Q, Yao H, Wu D, Chen S, Sun A: A BCR-JAK2 fusion gene from ins $(22 ; 9)(q 11 ; p 13 p 24)$ in a patient with atypical chronic myeloid leukemia. Leuk Lymphoma 2013, 54:2322-2324.

12. Wang ES, Maslak P, Cathcart K, Jurcic JG: Acute myeloid leukemia with $\mathrm{t}(5 ; 18)(\mathrm{q} 35 ; \mathrm{q} 21)$. Cancer Genet Cytogenet 2001, 127:71-73.

13. Daraki A, Bourantas LK, Manola KN: Translocation $\mathrm{t}(5 ; 18)(\mathrm{q} 35 ; \mathrm{q} 21)$ as a rare nonrandom abnormality in acute myeloid leukemia. Cytogenet Genome Res 2013, 139:289-294.

14. Fathi AT, Chen YB: Treatment of FLT3-ITD acute myeloid leukemia. Am J Blood Res 2011, 1:175-189.

15. Santos FP, Jones D, Qiao W, Cortes JE, Ravandi F, Estey EE, Verma D, Kantarjian H, Borthakur G: Prognostic value of FLT3 mutations among different cytogenetic subgroups in acute myeloid leukemia. Cancer 2011, 117:2145-2155.

16. Schiller J, Praulich I, Krings Rocha C, Kreuzer KA: Patient-specific analysis of FLT3 internal tandem duplications for the prognostication and monitoring of acute myeloid leukemia. Eur J Haematol 2012, 89:53-62.

17. Thanasopoulou A, Tzankov A, Schwaller J: Potent cooperation between the NUP98-NSD1 fusion and FLT3-ITD mutation in acute myeloid leukemia induction. Haematologica 2014, 99:1465-1471.

18. Kolomietz E, Al-Maghrabi J, Brennan S, Karaskova J, Minkin S, Lipton J, Squire JA: Primary chromosomal rearrangements of leukemia are frequently accompanied by extensive submicroscopic deletions and may lead to altered prognosis. Blood 2001, 97:3581-3588.

19. Bacher U, Schnittger S, Kern W, Hiddemann W, Haferlach T, Schoch C: The incidence of submicroscopic deletions in reciprocal translocations is similar in acute myeloid leukemia, BCR-ABL positive acute lymphoblastic leukemia, and chronic myeloid leukemia. Haematologica 2005 90:558-559.

20. Moon HW, Chang YH, Kim TY, Oh BR, Min HC, Kim BK, Ahn HS, Cho HI, Lee DS: Incidence of submicroscopic deletions vary according to disease entities and chromosomal translocations in hematologic malignancies: investigation by fluorescence in situ hybridization. Cancer Genet Cytogenet 2007, 175:166-168.

21. Dolan M, Peterson B, Hirsch B: Array-based comparative genomic hybridization characterizes a deletion associated with a $t(15 ; 17)$ in acute promyelocytic leukemia. Am J Clin Pathol 2008, 130:818-823.

22. Goodarzi AA, Jeggo PA: The repair and signaling responses to DNA double-strand breaks. Adv Genet 2013, 82:1-45.

23. Johansson B, Harrison CJ: Acute myeloid leukemia. In Cancer Cytogenetics. Edited by Heim S, Mitelman F. New Jersey: John Wiley \& Sons, Inc., Hoboken; 2009:45-139.

24. Morris SW, Kirstein MN, Valentine MB, Dittmer KG, Shapiro DN, Saltman DL, Look AT: Fusion of a kinase gene, ALK, to a nucleolar protein gene, NPM, in non-Hodgkin's lymphoma. Science 1994, 263:1281-1284

25. Redner RL, Rush EA, Faas S, Rudert WA, Corey SJ: The $t(5 ; 17)$ variant of acute promyelocytic leukemia expresses a nucleophosmin-retinoic acid receptor fusion. Blood 1996, 87:882-886.

26. Jaju RJ, Fidler C, Haas OA, Strickson AJ, Watkins F, Clark K, Cross NC, Cheng JF, Aplan PD, Kearney L, Boultwood J, Wainscoat JS: A novel gene, NSD1, is fused to NUP98 in the $t(5 ; 11)(q 35 ; p 15.5)$ in de novo childhood acute myeloid leukemia. Blood 2001, 98:1264-1267.

27. Itoh M, Okazaki T, Tashima M, Sawada H, Uchiyama T: Acute myeloid leukemia with $\mathrm{t}(5 ; 11)$ : two case reports. Leuk Res 1999, 23:677-680.

28. Thiede C, Koch S, Creutzig E, Steudel C, Illmer T, Schaich M, Ehninger G: Prevalence and prognostic impact of NPM1 mutations in 1485 adult patients with acute myeloid leukemia (AML). Blood 2006, 107:4011-4020.

29. Trimarchi T, Ntziachristos $P$, Aifantis I: A new player SETs in myeloid malignancy. Nat Genet 2013, 45:846-847.

30. Albano F, Anelli L, Zagaria A, Coccaro N, Casieri P, Minervini A, Specchia G: SETBP1 and miR_4319 dysregulation in primary myelofibrosis progression to acute myeloid leukemia. J Hematol Oncol 2012, 5:48.
31. Cristobal I, Blanco FJ, Garcia-Orti L, Marcotegui N, Vicente C, Rifon J, Novo FJ, Bandres E, Calasanz MJ, Bernabeu C, Odero MD: SETBP1 overexpression is a novel leukemogenic mechanism that predicts adverse outcome in elderly patients with acute myeloid leukemia. Blood 2010, 115:615-625.

32. Goshima G, Mayer M, Zhang N, Stuurman N, Vale RD: Augmin: a protein complex required for centrosome-independent microtubule generation within the spindle. J Cell Biol 2008, 181:421-429.

33. Fuchs $\mathrm{O}$ : Important genes in the pathogenesis of $5 q$ - syndrome and their connection with ribosomal stress and the innate immune system pathway. Leuk Res Treat 2012, 2012:179402.

34. Eisenmann KM, Dykema KJ, Matheson SF, Kent NF, DeWard AD, West RA, Tibes R, Furge KA, Alberts AS: 5q- myelodysplastic syndromes: chromosome $5 q$ genes direct a tumor-suppression network sensing actin dynamics. Oncogene 2009, 28:3429-3441.

35. Douet-Guilbert N, De Braekeleer E, Basinko A, Herry A, Gueganic N, Bovo C, Trillet K, Dos Santos A, Le Bris MJ, Morel F, Eveillard JR, Berthou C, De Braekeleer M: Molecular characterization of deletions of the long arm of chromosome 5 (del(5q)) in 94 MDS/AML patients. Leukemia 2012, 26:1695-1697.

36. Zhao N, Stoffel A, Wang PW, Eisenbart JD, Espinosa R 3rd, Larson RA, Le Beau MM: Molecular delineation of the smallest commonly deleted region of chromosome 5 in malignant myeloid diseases to 1-1.5 Mb and preparation of a PAC-based physical map. Proc Natl Acad Sci U S A 1997, 94:6948-6953.

37. Jerez A, Gondek LP, Jankowska AM, Makishima H, Przychodzen B, Tiu RV O'Keefe CL, Mohamedali AM, Batista D, Sekeres MA, McDevitt MA, Mufti GJ, Maciejewski JP: Topography, clinical, and genomic correlates of $5 q$ myeloid malignancies revisited. J Clin Oncol 2012, 30:1343-1349.

38. Stoddart A, Fernald AA, Wang J, Davis EM, Karrison T, Anastasi J, Le Beau MM: Haploinsufficiency of del(5q) genes, Egr1 and Apc, cooperate with Tp53 loss to induce acute myeloid leukemia in mice. Blood 2014, 123:1069-1078.

39. Ebert BL: Molecular dissection of the $5 \mathrm{q}$ deletion in myelodysplastic syndrome. Semin Oncol 2011, 38:621-626.

40. Fu CT, Zhu KY, Mi JQ, Liu YF, Murray ST, Fu YF, Ren CG, Dong ZW, Liu YJ, Dong $M$, Jin $Y$, Chen Y, Deng M, Zhang W, Chen B, Breslin P, Chen SJ, Chen Z, Becker MW, Zhu J, Zhang JW, Liu TX: An evolutionarily conserved PTEN-C/EBPalpha-CTNNA1 axis controls myeloid development and transformation. Blood 2010, 115:4715-4724.

41. Matsumura K, Sakai C, Kawakami S, Yamashita F, Hashida M: Inhibition of cancer cell growth by GRP78 siRNA lipoplex via activation of unfolded protein response. Biol Pharm Bull 2014, 37:648-653.

42. Richardson PG, Schlossman R, Hideshima T, Anderson KC: New treatments for multiple myeloma. Oncology 2005, 19:1781-1792. discussion 1792, 1795-1787

43. O'Connor OA, Wright J, Moskowitz C, Muzzy J, MacGregor-Cortelli B, Stubblefield M, Straus D, Portlock C, Hamlin P, Choi E, Dumetrescu O, Esseltine D, Trehu E, Adams J, Schenkein D, Zelenetz AD: Phase II clinical experience with the novel proteasome inhibitor bortezomib in patients with indolent non-Hodgkin's lymphoma and mantle cell lymphoma. J Clin Oncol 2005, 23:676-684.

44. Terpos E, Verrou E, Banti A, Kaloutsi V, Lazaridou A, Zervas K: Bortezomib is an effective agent for MDS/MPD syndrome with $5 q$ - anomaly and thrombocytosis. Leuk Res 2007, 31:559-562.

45. Shao L, Kang SH, Li J, Hixson P, Taylor J, Yatsenko SA, Shaw CA, Milosavljevic A, Chang CC, Cheung SW, Patel A: Array comparative genomic hybridization detects chromosomal abnormalities in hematological cancers that are not detected by conventional cytogenetics. J Mol Diagn 2010, 12:670-679.

46. Walter MJ, Payton JE, Ries RE, Shannon WD, Deshmukh H, Zhao Y, Baty J, Heath S, Westervelt P, Watson MA, Tomasson MH, Nagarajan R, O'Gara BP, Bloomfield CD, Mrozek K, Selzer RR, Richmond TA, Kitzman J, Geoghegan J, Eis PS, Maupin R, Fulton RS, McLellan M, Wilson RK, Mardis ER, Link DC, Graubert TA, DiPersio JF, Ley TJ: Acquired copy number alterations in adult acute myeloid leukemia genomes. Proc Natl Acad Sci U S A 2009, 106:12950-12955.

47. Bullinger L, Kronke J, Schon C, Radtke I, Urlbauer K, Botzenhardt U, Gaidzik V, Cario A, Senger C, Schlenk RF, Downing JR, Holzmann K, Dohner K, Dohner $\mathrm{H}$ : Identification of acquired copy number alterations and uniparental disomies in cytogenetically normal acute myeloid leukemia 
using high-resolution single-nucleotide polymorphism analysis. Leukemia 2010, 24:438-449.

48. Kjeldsen E, Roug AS: A novel unbalanced de novo translocation der(5)t $(4 ; 5)(q 26 ; q 21.1)$ in adult T-cell precursor lymphoblastic leukemia. Mol Cytogenet 2012, 5:21.

49. ISCN: An International System for Human Cytogenetic Nomenclature (2013). Basel: S. Karger and Cytogenetic and Genome Research; 2013.

doi:10.1186/s13039-014-0063-x

Cite this article as: Kjeldsen: A novel insertion ins(18;5)(q21.1;

q31.2q35.1) in acute myeloid leukemia associated with microdeletions

at $5 q 31.2,5 q 35.1 q 35.2$ and $18 q 12.3 q 21.1$ detected by oligobased array

comparative genomic hybridization. Molecular Cytogenetics 2014 7:63.

\section{Submit your next manuscript to BioMed Central and take full advantage of:}

- Convenient online submission

- Thorough peer review

- No space constraints or color figure charges

- Immediate publication on acceptance

- Inclusion in PubMed, CAS, Scopus and Google Scholar

- Research which is freely available for redistribution 